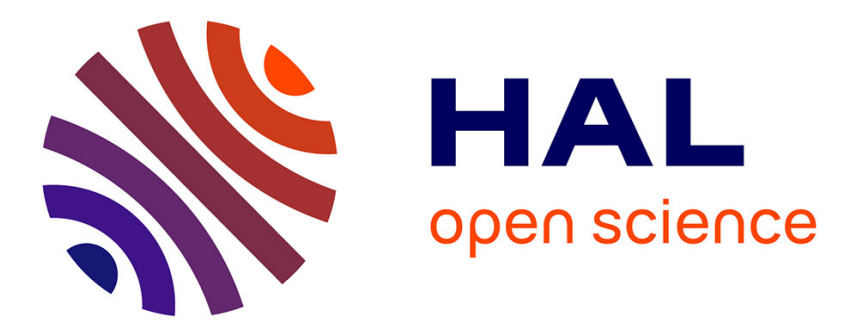

\title{
Potentials of CRISPR in liver research and therapy
}

Robin Loesch, Christèle Desbois-Mouthon, Sabine Colnot

\section{To cite this version:}

Robin Loesch, Christèle Desbois-Mouthon, Sabine Colnot. Potentials of CRISPR in liver research and therapy. Clinics and Research in Hepatology and Gastroenterology, 2019, 43, pp.5 - 11. 10.1016/j.clinre.2018.05.001 . hal-03484597

\section{HAL Id: hal-03484597 \\ https://hal.science/hal-03484597}

Submitted on 20 Dec 2021

HAL is a multi-disciplinary open access archive for the deposit and dissemination of scientific research documents, whether they are published or not. The documents may come from teaching and research institutions in France or abroad, or from public or private research centers.
L'archive ouverte pluridisciplinaire $\mathbf{H A L}$, est destinée au dépôt et à la diffusion de documents scientifiques de niveau recherche, publiés ou non, émanant des établissements d'enseignement et de recherche français ou étrangers, des laboratoires publics ou privés.

\section{(ㄷ)(1) $\$$}

Distributed under a Creative Commons Attribution - NonCommerciall 4.0 International 


\section{Potentials of CRISPR in liver research and therapy}

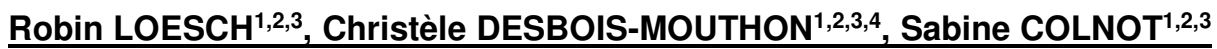

1 INSERM, U1016, Institut Cochin, Paris, 75014, France

${ }^{2}$ CNRS, UMR8104, Paris, 75014, France

${ }^{3}$ Université Paris Descartes, Sorbonne Paris Cité, Paris, 75006, France

${ }^{4}$ Sorbonne Université, INSERM, Saint-Antoine Research Center, Paris, 75012, France

\section{Corresponding author:}

\section{Dr Sabine Colnot,}

Institut Cochin INSERM U1016

24, rue du Faubourg Saint Jacques

75014 PARIS

FRANCE

Tel: +33144412567

E-mail : sabine.colnot@inserm.fr

Suggested reviewers:

Diego Francesco CALVISI

Email: calvisid@uniss.it

Anne DUBART-KUPPERSCHMITT

Email: anne.dubart@inserm.fr 


\section{Potentials of CRISPR in liver research and therapy}

\section{Summary}

The CRISPR technology is rapidly transforming the field of liver research by its versatility and easy use. In vivo gene editing of hepatocytes in adult mice can be achieved using a broad toolbox for both fundamental research and development of therapeutic strategies for future clinical applications. Recent studies showed that CRISPR has a real potential to treat hereditary liver diseases as well as virally induced pathologies. This short review recapitulates very recent advancements regarding the use of CRISPR in liver research and therapy.

Key words : CRISPR, liver, in vivo, research ,therapy 


\section{Introduction}

The CRISPR locus, for Cluster Regulatory Interspaced Short Palindromic Repeats, was discovered in Escherichia Coli in 1987 (1) before being found in about $40 \%$ of the bacteria and $90 \%$ of the archaea. The CRISPR system rapidly appeared as a defense mechanism against foreign genetic material via small guide RNA (gRNA) targeted DNA breaks (2). This mechanism is now used as a powerful gene editing tool notably in human cells (3) (4). It induces precise double-stranded DNA breaks which allows specific gene editing through the well-known DNA repair mechanisms. Among the six major types of CRISPR mechanisms (5), the type II CRISPR/Cas9 system is largely used and relies one a single Cas9 nuclease and two small RNAs, the CRISPR RNA (crRNA) and the trans-activating crRNA (tracrRNA). The crRNA encodes a gRNA complementary to the targeted DNA. Rapid design of the gRNA based on the targeted sequence followed by cloning into the crRNA backbone allows a cheap and rapid setup of the method compared to less friendly and more expensive Transcription activator-like effector nucleases (TALENs) or Zinc Finger Nucleases (ZFN). Since the simplification of the use of CRISPR/Cas9 mechanism from Staphylococcus Pyogenes (SpCas9) via artificial fusion of the crRNA and the tracrRNA into a single guide RNA (sgRNA), the scientific community started to wildly use the CRISPR/SpCas9 technology at the expense of TALENS or ZFN.

Most CRISPR-mediated gene editing possibilities flow from the capability of the cells to repair double-strand breaks (DSBs). DSBs are repaired using two distinct mechanisms, Non-Homologous End Joining (NHEJ) or Homology Directed Repair (HDR). NHEJ induces insertions and deletions (indels) which favor gene disruption by making frameshifts or targeted large deletions using multiple sgRNA $(6,7)$. It is also important to mention that alternative NHEJ repair (8) can be exploited to perform targeted DNA insertion (9). HDR uses the homologous recombination pathway. In this case, DSBs repair using a custom exogenous DNA template containing the genetic modification allows precise gene engineering (Figure 1). Similarly, but through a not yet fully understand mechanism, the same outcome can be achieved using a single-stranded DNA template.

The very versatile CRISPR/Cas9 gene editing tool became a broad toolbox with different kinds of applications like genome engineering, RNA targeting (10), transcriptional regulation (11) or genome loci localization (12). The study and improvement of CRISPR technology became by itself a topic of research that is continually building the future tools for research and clinical applications. The potential of CRISPR in therapeutics immediately started to fuel highly ambitious dreams but its use has facilitated first cancer and fundamental researches. 


\section{CRISPR in liver research}

For several years, in vivo genetic alteration can be induced using the well-known CRELOX system. It uses the property of the Cre recombinase to catalyze sequence specific recombination. Tissue-specific recombination can be achieved by putting the recombinase under the control of a tissue-specific promoter. In addition, time control of the genetic modification is achieved using a Cre recombinase fused with the DNA binding domain of the human estrogen receptor mutated to specifically bind tamoxifen and consequently migrate to the nucleus. This system is reliable but requires expensive and time consuming mouse transgenesis and breeding. As an alternative, liver targeting in adult mouse can be achieved using hydrodynamic tail vein injection (HTVI) $(\mathbf{1 3}, \mathbf{1 4})$. HTVI consists in rapid tail vein injection of a large volume of a plasmid DNA solution which allows hepatocyte transfection through a mechanism called hydroporation. Importantly, this technique remains invasive and associated with possible mice mortality as it induces a transient irregularity of heart function, sharp increase in venous pressure, enlargement of liver fenestrae and enhancement of hepatocyte membrane permeability.

The use of CRISPR/Cas9 rapidly became a technique of choice to target the liver in vivo (Figure 2a). One of the first study reporting the use of the CRISPR/Cas9 system in vivo to target the mouse liver was reported in 2014 (15). This study described the delivery of CRISPR plasmids using HTVI to target cancer genes. The strength of the paper is the demonstration that CRISPR/Cas9 can be used to perform different gene editing events in vivo. Direct loss of function (LOF) of common tumor suppressor genes (TSGs), p53 and Pten, both alone or in combination, was performed using bi-allelic indels-mediated frameshifts. Going deeper into precise gene editing, the authors were also able to insert activating point mutations into Ctnnb1, the gene encoding $\beta$-catenin via recombination using a single-stranded DNA template co-injected with the CRISPR plasmid. Even though this technique was poorly efficient, this work highlighted the possibility to disrupt any genes in the liver, bypassing the need for engineer embryonic stem cells and breeding while remaining sufficient to promote and study liver carcinogenesis.

Combining CRISPR/Cas9 and HTVI with integrative transposon like Piggy Bac (PB) and Sleeping Beauty (SB) allowed cell lineage analysis and the development of in vivo genetic screens $(16,17)$. PB and $\mathrm{SB}$ are transposons that need a transposase for genome integration. Co-injection in mice of a vector carrying sgRNA and Cas9 expression cassettes flanked by SB or PB inverted repeats (CRISPR vector) and a vector encoding the transposase allows uncontrolled insertion of the CRISPR vector in the genome of transfected cells. Using a sgRNA library and a PB transposon, Xu et al(18) showed that it is possible to target thousands of genes at once to screen for TSGs. After HTVI of the PB library and tumor development, the analysis of the tumor genome reveals causal genetic alterations hence the potential tumorigenic effect of new TSGs in hepatocellular carcinoma ( $\mathrm{HCC}$ ). 
Cancer cells are mostly the consequences of synergistic mutations rather than unique driver ones. Weber et al (19) used a similar approach to $\mathrm{Xu}$ and colleagues to screen in mice for synergistic mutations in liver cancers. Using a SB-based methodology, they performed a somatic multiplex-mutagenesis by targeting ten TSGs frequently mutated or epigenetically silenced in intrahepatic cholangiocarcinoma or HCC. Once again, the analysis of the developing tumors revealed positively selected mutations like alteration of Pten, Tet2 or Apc which are found mutated in human HCC. Interestingly, mutations of other cancer genes like BRCA1 and 2 were largely absent in $\mathrm{HCC}$ which is consistent with genome wide analysis of human HCCs. To go deeper into the understanding of HCCs development, one of the perspectives using CRISPR is to induce co-occurring mutations revealed by whole genome analysis of human tumors (20). Such advancement would allow the development of relevant pre-clinical cancer models.

In 2015, Zhang's team reported a huge breakthrough regarding in vivo targeting of adult mouse liver. The characterization of six smaller Cas9 orthologues shed lights on the equally efficient and shorter Cas9 from Staphylococcus Aureus (SaCas9) (21). The smaller size of the SaCas9 coding sequence allows its packaging into a single adeno-associated virus (AAV) along with the sgRNA expression cassette. The specific targeting of the liver can be achieved using the thyroxin-binding globulin promoter to drive the SaCas9 expression (22). Hepatic targeting is reinforced by the production of hepatotropic AAV particles harboring serotype 8 protein capsids (23). Using this technology, Zhang's team targeted the proprotein convertase subtilisin/kexin type 9 (PCSK9), involved in cholesterol homeostasis. They obtained more than $40 \%$ of indels at the targeted locus on total liver one week after AAV injection. In addition, the reduction of serum PCSK9 protein level was correlated with a diminution of total cholesterol level.

Recently, a multiplexing approach was reported using $A A V$ vectors to screen the tumorigenic effect of recurrently mutated genes (RMGs) in human cancers (24). A library of sgRNA targeting the RMGs was built according to pan-cancer TCGA data and cloned into a base vector containing the sgRNA cassette as well as a Cre expression cassette. To facilitate tumorigenesis, a cassette coding a sgRNA targeting P53 was included into the base vector. Intravenous injection of the AAV vector library (AAV-CRISPR mTSG library) in a mouse model which possesses a Cre inducible Cas9 coupled with GFP allowed the concomitant targeting of P53 and multiple genes depending on the transduced AAV. Each mouse receiving the AAVCRISPR mTSG library died within 4 months due to tumor development. Sequencing of the targeted sites in the tumors shed light on their mutational landscape which corresponded to the positively selected mutations driving tumorigenesis. Genome analysis of 37 tumor samples permited to bring out potential oncogenic cooperations for which further investigation was initiated by inducing single mutation in a P53-sensitized background.

As demonstrated above the CRISPR technology can easily be combined with already existing CRELOX mouse models and used for sequential LOF (Figure 2b). The ease with which new mouse models can be developed using this approach already makes it a broadly used method. 


\section{CRISPR potential in liver targeted therapy}

By targeting the cholesterol metabolism, Zhang's laboratory illustrated what could be the future of gene editing-mediated gene therapy (GT)(21). Liver diseases are interesting candidates for GT as many of them are due to mutations in a single gene and few have already been targeted in mouse using CRISPR like ornithine transcarbamylase deficiency $(25)$, tyrosinemia $(7,26)$ or hemophilia as discussed in the following section. Boosted by the development of non-invasive delivery methods, successful strategies targeting liver disorders increased over the past four years (Figure 2c). Following examples of recent achievements describe the potential of CRIPSR/Cas9 technology in liver diseases.

\section{Hemophilia A and B:}

Hemophilia $A$ and $B$ are recessive $X$-linked coagulation disorders due to mutations in the genes encoding the clotting factor VIII (FVIII) or IX (FIX) respectively and affect about 400,000 individuals worldwide (27). The monogenic aspect of these bleeding disorders made them very attractive for GT. Different strategies were used to supply patients with functional FVIII or FIX. Currently, protein substitution therapy using recombinant protein or plasma-derived clotting factor improves life quality and expectancy of patients. Unfortunately, this prophylaxis treatment requires expensive and repetitive administration of FVIII or FIX against which patients can develop neutralizing antibodies. Development of curative treatments via GT are needed to improve life quality of hemophilia patients. In this setting, clinical trials of GT using AAV to provide the missing clotting factor are promising (28). However, AAV-mediated transgene expression is long but not permanent. The development of CRISPR/Cas9-mediated gene editing raised the possibility to directly correct mutations in hemophilia patient hepatocytes.

mice $(\mathbf{2 9}, \mathbf{3 0})$. Using the previously described HTVI delivery system Huai et al used a Cas9-sgRNA expression vector along with a self-linearizable double-stranded donor template to correct an eight nucleotides-long deletion in FIX exon 8. They were able to induce targeted HDR and reached more than $1 \%$ of correction which was sufficient to restore homeostasis. More recently, Ohmori et al (30), similarly to Zhang's strategy, made use of the previously described SaCas9 driven by an hepatic promoter delivered along with the sgRNA using AAV vectors to in vivo target the mutated FIX. Co-injection of an AAV carrying the CRISPR components along with an AAV containing the corrected sequence successfully induced HDR and correction of a twelve base-pair deletion in the FIX exon 8, reversing the phenotype of 0,14 and 182 days old hemophilia mice.

The main hurdle performing in vivo gene editing is safety concern due to potential off-target effects of the CRISPR/Cas9 system. The use of induced pluripotent stem cells (iPSC) would allow a control over the editing and the possible mutational effect of CRISPR. Recently, a Chinese group reported the use of hemophilia B patient-derived iPSC (31) to correct a missense mutation (c.676 C>T) in the FIX gene using HDR after transient transfection of a plasmid encoding CRISPR/Cas9 system and a single-stranded oligonucleotide bearing the correction. The high efficiency (10 corrected clones and 38 clones witnessing 
efficient cleavage over 45 sequenced) and the absence of off-target effect at potential anticipated site reinforced the notion that CRISPR/Cas9 can be simultaneously efficient and precise. The corrected iPSC derived into hepatocytes were found to efficiently secrete FIX. This personalized PSC-based GT bypasses the off-target problematic as a deep analysis at the cell scale can be performed before possible reinjection to human but it comes with every safety questions regarding the use of iPSC-derived cells.

\section{Hepatitis:}

Hepatitis $B$ and $C$ viruses (HBV/HCV) are pathogens associated to a higher risk of cirrhosis and HCC. Thus, $78 \%$ of liver cancers arise in patients with HBV or HCV chronic infections (32). As HCC is the second cause of cancer-related death worldwide (33), fighting HBV and HCV chronic infections would decrease the incidence of HCC hence the related mortality. In the case of HBV, persistence of episomal HBV genome known as the covalently closed circular DNA (cccDNA) is responsible for the virion production and chronic infection (34). Current therapies targeting HBV are based on reverse transcriptase inhibitors or immunomodulation treatments which are prone to drug resistance and/or accompanied with side effects. Most of all, the treatments and post infection vaccination failed to eradicate the cccDNA. Consequently, resurgence of HBV replication occurs upon treatment withdrawal. Regarding HCV, no preventive vaccine is available. Recently, treatment with different cocktails of protease inhibitors showed efficient and promising cure of HCV (35). Resistance to therapy of a subset of patients still illustrates the need for other ways to fight HCV infection (36).

Targeting the HBV cccDNA would be a way to cure HBV. In 2017, Hao et al (37) presented the in vitro demonstration that CRISPR/Cas9 can provide a radical cure for inserted HBV genome. The authors used a HepG2 cell line expressing both HBV DNA and protein surface due to HBV genome integration. After transient transfection of SpCas9 along with one sgRNA targeting the repeats flanking the HBV genome, they detected the 3,173 bp deletion of the HBV genome integrated.

A couple of studies tried to disrupt the HBV genome in vivo. In 2014, Lin et al (38) used HTVI to inject both the HBV genome-containing plasmid and a CRISPR/Cas9 plasmid targeting sequences highly conserved in HBV genotype A, B and C. Lower amounts of hepatitis $B$ antigen surface were found in the serum of injected mice as well as lower intrahepatic HBV-expressing vector showing the potential of CRIPSR to cleave HBV genome and increase HBV clearance. Very recently, another Chinese group (39) used the previously described AAV-mediated delivery of the CRISPR/Cas9 technology to target the HBV genome. They used a murine model in which persistent HBV replication was achieved for several months after HTVI of PAAV/HBV1.2 plasmids. Efficient targeting of the HBV genome was observed but a nonsignificant decrease of hepatitis $B$ surface antigen and HBV DNA were observed after a secondary and higher AAV titer injection. One can ask if the decrease of HBV surface antigen and HBV DNA observed after the second injection of $A A V$ could be due to an immune response relative to a second exposition to the AAV8 vector which could induce the elimination of cells carrying the AAV/HBV1.2. This study reveals the potential of AAV-mediated delivery of CRISPR/Cas9 to target HBV genome in vivo but also shows that 
further improvements need to be made before testing in humans. In vivo targeting of the HBV genome using AAV delivery of the CRISPR/Cas9 is an encouraging approach that needs to overcome challenging issues which are described in details elsewhere (40-42). Concerning the RNA genome of HCV, only one in vitro study performed in Huh-7.5 cells using a Cas9 targeting RNA (FnCas9) showed the possibility to inhibit HCV expression suggesting the potential in targeting RNA virus using CRISPR (43).

\section{Conclusion}

If the CRISPR technology has revolutionized gene editing and research in general, the combination of CRISPR with AAV vectors is close to revolutionize in vivo liver research in mouse models. The less immunogenic, non-integrative and high hepatotropic features of the AAV8 make it attractive and efficient to target the liver. AAV vectors are progressively replacing both the use of efficient but more inflammatory adenoviruses and the more invasive and less efficient HTVI which remains highly attractive due to its inexpensiveness. If HTVI combined with the integrative feature of the sleeping beauty or piggy bac transposon allows the easy setup of in vivo screens for new TSGs, Wang et al (24) showed that AAV vectors could be used as well.

Regarding the use of CRISPR in clinics, the two major issues reside in the delivery method and the potential off-target effects. Nowadays, GT strategies rely on the use of viral vectors. It is commonly thought that $A A V$ vectors allow transgene expression from an episomal template avoiding insertional oncogenesis which make them very attractive for gene delivery. However, it is important to notice that clonal integration of AAV2 inducing oncogene overexpression has been reported in HCC (44). More recently, frequent small integration of AAV8 inverted terminal repeats (ITRs) at the targeted site has been reported which illustrates the recombinogenic properties of ITRs (45). However, analysis of liver biopsies from patients under clinical trials using AAV suggests that such events are rare and may not pose a major risk of cancer (46). Another concern is vector-induced liver inflammation. Widespread exposure to numerous AAV variants and serotypes within the human population favors the development of immune response against AAV vector protein capsid reducing delivery efficacy and inducing liver inflammation. While huge progresses are being made to engineer less immunogenic AAV protein capsid (47), non-viral delivery methods using nanoparticle

are explored to enhance the safety of liver GT $(26,48,49)$. Concerning the CRISPR off-target effects, it is now accepted that the amount of off targets is linked to both the amount of CRISPR effectors in the cells and the time of cell exposure. AAV vectors allow long lasting expression of the CRISPR machinery and we still have no feedback on potential impact of CRISPR long term expression at a human scale. In these regards, transient delivery of CRISPR using non-viral methods is attractive and must be seriously considered. 
1. Ishino Y, Shinagawa H, Makino K, Amemura M, Nakata A. Nucleotide sequence of the iap gene, responsible for alkaline phosphatase isozyme conversion in Escherichia coli, and identification of the gene product. J Bacteriol 1987;169:5429-5433.

2. Jinek M, Chylinski K, Fonfara I, Hauer M, Doudna JA, Charpentier E. A programmable dual-RNAguided DNA endonuclease in adaptive bacterial immunity. Science 2012;337:816-821.

3. Ran FA, Hsu PD, Wright J, Agarwala V, Scott DA, Zhang F. Genome engineering using the CRISPRCas9 system. Nat Protoc 2013;8:2281-2308.

4. Mali P, Yang L, Esvelt KM, Aach J, Guell M, DiCarlo JE, Norville JE, et al. RNA-guided human genome engineering via Cas9. Science 2013;339:823-826.

5. Wright AV, Nunez JK, Doudna JA. Biology and Applications of CRISPR Systems: Harnessing Nature's Toolbox for Genome Engineering. Cell 2016;164:29-44.

6. Engelholm LH, Riaz A, Serra D, Dagnaes-Hansen F, Johansen JV, Santoni-Rugiu E, Hansen SH, et al. CRISPR/Cas9 Engineering of Adult Mouse Liver Demonstrates That the Dnajb1-Prkaca Gene Fusion Is Sufficient to Induce Tumors Resembling Fibrolamellar Hepatocellular Carcinoma. Gastroenterology 2017;153:1662-1673 e1610.

7. Pankowicz FP, Barzi M, Legras X, Hubert L, Mi T, Tomolonis JA, Ravishankar M, et al. Reprogramming metabolic pathways in vivo with CRISPR/Cas9 genome editing to treat hereditary tyrosinaemia. Nat Commun 2016;7:12642.

8. Seol JH, Shim EY, Lee SE. Microhomology-mediated end joining: Good, bad and ugly. Mutat Res 2017.

9. Yao X, Wang X, Liu J, Hu X, Shi L, Shen X, Ying W, et al. CRISPR/Cas9 - Mediated Precise Targeted Integration In Vivo Using a Double Cut Donor with Short Homology Arms. EBioMedicine 2017;20:19-26.

10. Abudayyeh OO, Gootenberg JS, Essletzbichler P, Han S, Joung J, Belanto JJ, Verdine V, et al. RNA targeting with CRISPR-Cas13. Nature 2017;550:280-284.

11. Perez-Pinera P, Kocak DD, Vockley CM, Adler AF, Kabadi AM, Polstein LR, Thakore PI, et al. RNAguided gene activation by CRISPR-Cas9-based transcription factors. Nat Methods 2013;10:973-976.

12. Ma H, Naseri A, Reyes-Gutierrez P, Wolfe SA, Zhang S, Pederson T. Multicolor CRISPR labeling of chromosomal loci in human cells. Proc Natl Acad Sci U S A 2015;112:3002-3007.

13. Zhang G, Gao X, Song YK, Vollmer R, Stolz DB, Gasiorowski JZ, Dean DA, et al. Hydroporation as the mechanism of hydrodynamic delivery. Gene Ther 2004;11:675-682.

14. Chen X, Calvisi DF. Hydrodynamic transfection for generation of novel mouse models for liver cancer research. Am J Pathol 2014;184:912-923.

15. Xue W, Chen S, Yin H, Tammela T, Papagiannakopoulos T, Joshi NS, Cai W, et al. CRISPR-mediated direct mutation of cancer genes in the mouse liver. Nature 2014;514:380-384.

16. Narayanavari SA, Chilkunda SS, Ivics Z, Izsvak Z. Sleeping Beauty transposition: from biology to applications. Crit Rev Biochem Mol Biol 2017;52:18-44.

17. Zhao S, Jiang E, Chen S, Gu Y, Shangguan AJ, Lv T, Luo L, et al. PiggyBac transposon vectors: the tools of the human gene encoding. Transl Lung Cancer Res 2016;5:120-125.

18. Xu C, Qi X, Du X, Zou H, Gao F, Feng T, Lu H, et al. piggyBac mediates efficient in vivo CRISPR library screening for tumorigenesis in mice. Proc Natl Acad Sci U S A 2017;114:722-727.

19. Weber J, Ollinger R, Friedrich M, Ehmer U, Barenboim M, Steiger K, Heid I, et al. CRISPR/Cas9 somatic multiplex-mutagenesis for high-throughput functional cancer genomics in mice. Proc Natl Acad Sci U S A 2015;112:13982-13987.

20. Guichard C, Amaddeo G, Imbeaud S, Ladeiro Y, Pelletier L, Maad IB, Calderaro J, et al. Integrated analysis of somatic mutations and focal copy-number changes identifies key genes and pathways in hepatocellular carcinoma. Nat Genet 2012;44:694-698.

21. Ran FA, Cong L, Yan WX, Scott DA, Gootenberg JS, Kriz AJ, Zetsche B, et al. In vivo genome editing using Staphylococcus aureus Cas9. Nature 2015;520:186-191. 
22. Yan Z, Yan H, Ou H. Human thyroxine binding globulin (TBG) promoter directs efficient and sustaining transgene expression in liver-specific pattern. Gene 2012;506:289-294.

23. Chen SJ, Johnston J, Sandhu A, Bish LT, Hovhannisyan R, Jno-Charles O, Sweeney HL, et al. Enhancing the utility of adeno-associated virus gene transfer through inducible tissue-specific expression. Hum Gene Ther Methods 2013;24:270-278.

24. Wang G, Chow RD, Ye L, Guzman CD, Dai X, Dong MB, Zhang F, et al. Mapping a functional cancer genome atlas of tumor suppressors in mouse liver using AAV-CRISPR-mediated direct in vivo screening. Sci Adv 2018;4:eaao5508.

25. Yang Y, Wang L, Bell P, McMenamin D, He Z, White J, Yu H, et al. A dual AAV system enables the Cas9-mediated correction of a metabolic liver disease in newborn mice. Nat Biotechnol 2016;34:334-338.

26. Yin $\mathrm{H}$, Song CQ, Dorkin JR, Zhu LJ, Li Y, Wu Q, Park A, et al. Therapeutic genome editing by combined viral and non-viral delivery of CRISPR system components in vivo. Nat Biotechnol 2016;34:328333.

27. VandenDriessche T, Chuah MK. Hemophilia Gene Therapy: Ready for Prime Time? Hum Gene Ther 2017;28:1013-1023.

28. Nathwani AC, Tuddenham EG, Rangarajan S, Rosales C, McIntosh J, Linch DC, Chowdary P, et al. Adenovirus-associated virus vector-mediated gene transfer in hemophilia B. N Engl J Med 2011;365:2357-2365.

29. Huai C, Jia C, Sun R, Xu P, Min T, Wang Q, Zheng C, et al. CRISPR/Cas9-mediated somatic and germline gene correction to restore hemostasis in hemophilia B mice. Hum Genet 2017;136:875-883.

30. Ohmori T, Nagao Y, Mizukami H, Sakata A, Muramatsu SI, Ozawa K, Tominaga SI, et al. CRISPR/Cas9-mediated genome editing via postnatal administration of AAV vector cures haemophilia $B$ mice. Sci Rep 2017;7:4159.

31. He Q, Wang HH, Cheng T, Yuan WP, Ma YP, Jiang YP, Ren ZH. Genetic Correction and Hepatic Differentiation of Hemophilia B-specific Human Induced Pluripotent Stem Cells. Chin Med Sci J 2017;32:135-144.

32. Perz JF, Armstrong GL, Farrington LA, Hutin YJ, Bell BP. The contributions of hepatitis B virus and hepatitis $C$ virus infections to cirrhosis and primary liver cancer worldwide. J Hepatol 2006;45:529-538.

33. Levrero M, Zucman-Rossi J. Mechanisms of HBV-induced hepatocellular carcinoma. J Hepatol 2016;64:S84-S101.

34. Ji M, Hu K. Recent advances in the study of hepatitis B virus covalently closed circular DNA. Virol Sin 2017;32:454-464.

35. Bourliere M, Gordon SC, Flamm SL, Cooper CL, Ramji A, Tong M, Ravendhran N, et al. Sofosbuvir, Velpatasvir, and Voxilaprevir for Previously Treated HCV Infection. N Engl J Med 2017;376:2134-2146.

36. Kim S, Han KH, Ahn SH. Hepatitis C Virus and Antiviral Drug Resistance. Gut Liver 2016;10:890895.

37. Li H, Sheng C, Wang S, Yang L, Liang Y, Huang Y, Liu H, et al. Removal of Integrated Hepatitis B Virus DNA Using CRISPR-Cas9. Front Cell Infect Microbiol 2017;7:91.

38. Lin SR, Yang HC, Kuo YT, Liu CJ, Yang TY, Sung KC, Lin YY, et al. The CRISPR/Cas9 System Facilitates Clearance of the Intrahepatic HBV Templates In Vivo. Mol Ther Nucleic Acids 2014;3:e186.

39. Liu Y, Zhao M, Gong M, Xu Y, Xie C, Deng H, Li X, et al. Inhibition of hepatitis B virus replication via HBV DNA cleavage by Cas9 from Staphylococcus aureus. Antiviral Res 2018;152:58-67.

40. Yang HC, Chen PJ. The potential and challenges of CRISPR-Cas in eradication of hepatitis B virus covalently closed circular DNA. Virus Res 2018;244:304-310.

41. Moyo B, Bloom K, Scott T, Ely A, Arbuthnot P. Advances with using CRISPR/Cas-mediated gene editing to treat infections with hepatitis B virus and hepatitis C virus. Virus Res 2018;244:311-320.

42. Aravalli RN, Steer CJ. CRISPR/Cas9 therapeutics for liver diseases. J Cell Biochem 2017. 
43. Price AA, Sampson TR, Ratner HK, Grakoui A, Weiss DS. Cas9-mediated targeting of viral RNA in eukaryotic cells. Proc Natl Acad Sci U S A 2015;112:6164-6169.

44. Nault JC, Datta S, Imbeaud S, Franconi A, Mallet M, Couchy G, Letouze E, et al. Recurrent AAV2related insertional mutagenesis in human hepatocellular carcinomas. Nat Genet 2015;47:1187-1193.

45. Jarrett KE, Lee CM, Yeh YH, Hsu RH, Gupta R, Zhang M, Rodriguez PJ, et al. Somatic genome editing with CRISPR/Cas9 generates and corrects a metabolic disease. Sci Rep 2017;7:44624.

46. Gil-Farina I, Fronza R, Kaeppel C, Lopez-Franco E, Ferreira V, D'Avola D, Benito A, et al. Recombinant AAV Integration Is Not Associated With Hepatic Genotoxicity in Nonhuman Primates and Patients. Mol Ther 2016;24:1100-1105.

47. Bartel M, Schaffer D, Buning H. Enhancing the Clinical Potential of AAV Vectors by Capsid Engineering to Evade Pre-Existing Immunity. Front Microbiol 2011;2:204.

48. Finn JD, Smith AR, Patel MC, Shaw L, Youniss MR, van Heteren J, Dirstine T, et al. A Single Administration of CRISPR/Cas9 Lipid Nanoparticles Achieves Robust and Persistent In Vivo Genome Editing. Cell Rep 2018;22:2227-2235.

49. Miller JB, Zhang S, Kos P, Xiong H, Zhou K, Perelman SS, Zhu H, et al. Non-Viral CRISPR/Cas Gene Editing In Vitro and In Vivo Enabled by Synthetic Nanoparticle Co-Delivery of Cas9 mRNA and sgRNA. Angew Chem Int Ed Engl 2017;56:1059-1063.

50. Liu Y, Qi X, Zeng Z, Wang L, Wang J, Zhang T, Xu Q, et al. CRISPR/Cas9-mediated p53 and Pten dual mutation accelerates hepatocarcinogenesis in adult hepatitis $B$ virus transgenic mice. Sci Rep 2017;7:2796.

51. Gao M, Liu D. CRISPR/Cas9-based Pten knock-out and Sleeping Beauty Transposon-mediated Nras knock-in induces hepatocellular carcinoma and hepatic lipid accumulation in mice. Cancer Biol Ther 2017;18:505-512.

52. Liang WC, Liang PP, Wong CW, Ng TB, Huang JJ, Zhang JF, Waye MM, et al. CRISPR/Cas9 Technology Targeting Fas Gene Protects Mice From Concanavalin-A Induced Fulminant Hepatic Failure. J Cell Biochem 2017;118:530-536. 


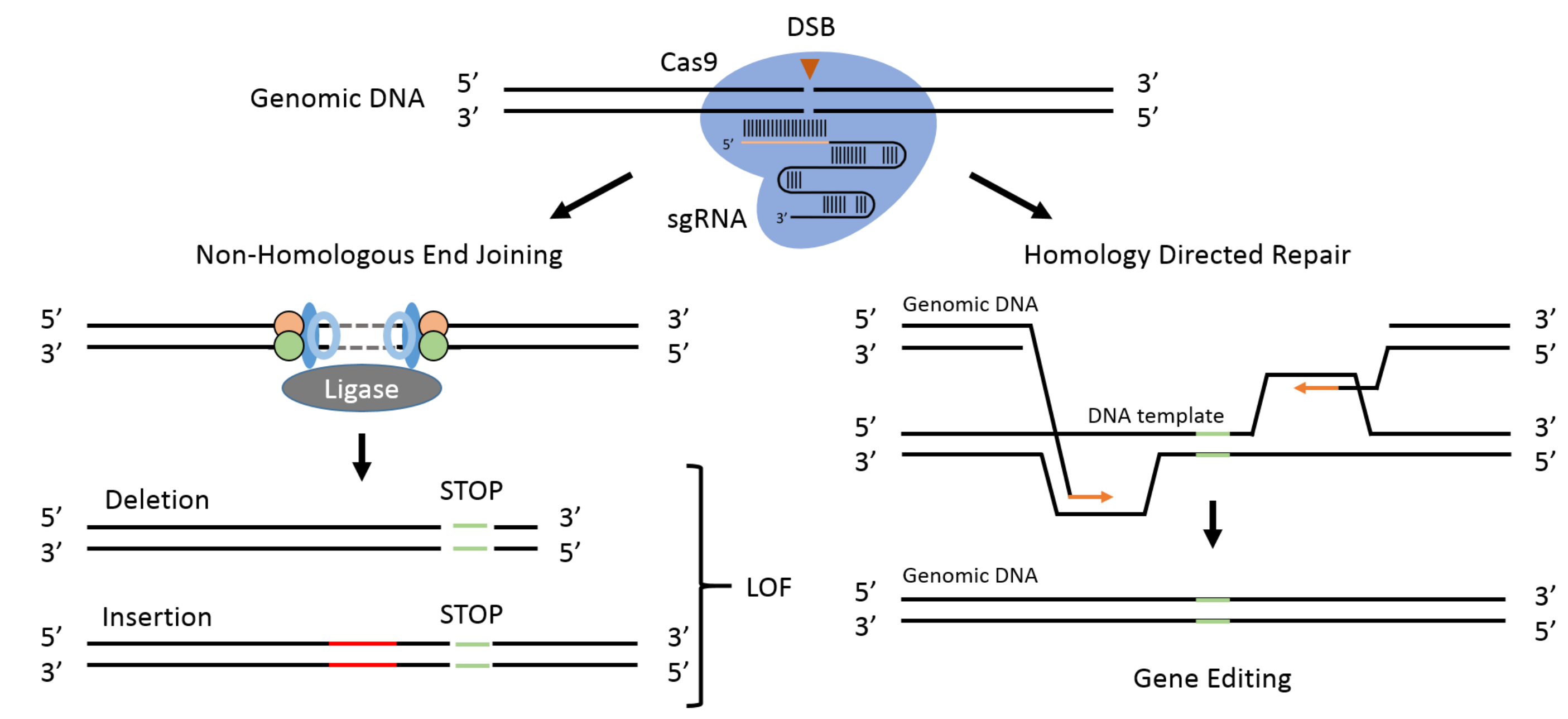

Figure 1: Gene editing using CRISPR. Upon CRISPR mediated double stranded break (DSB), the DNA repair mechanisms determine the outcome of the gene editing. The classical non-homologous end joining pathway is error prone and insertions and deletions resulting from the repair can induce frameshifts and premature STOP codons. Inversely the homology directed repair allow very precise gene editing. sgRNA : single guide RNA. 
In vivo gene editing toolbox

\begin{tabular}{|c|c|c|}
\hline $\begin{array}{l}\text { Delivery By IV } \\
\text { injection }\end{array}$ & Gene Editing & Highlights \\
\hline \multirow{2}{*}{$\begin{array}{c}\text { Adeno Associated } \\
\text { Virus }\end{array}$} & \multirow{2}{*}{$\begin{array}{c}\text { NHEJ }(21,39) \\
\operatorname{HDR}(25,30) \\
\text { Multiplexing (24) }\end{array}$} & Non integrative \\
\hline & & Sustained expression \\
\hline \multirow{3}{*}{$\begin{array}{l}\text { Hydrodynamic Tail } \\
\text { Vein Injection (9) }\end{array}$} & \multirow{3}{*}{$\begin{array}{c}\text { NHEJ }(6,7,15,50-52) \\
\operatorname{HDR}(9,15) \\
\text { Multiplexing }(18,19)\end{array}$} & Economical \\
\hline & & Combinable with SB/PB \\
\hline & & Invasive \\
\hline \multirow{3}{*}{ Lipid Nanoparticles } & \multirow{3}{*}{$\begin{array}{c}\operatorname{NHEJ}(48,49) \\
\operatorname{HDR}(26)\end{array}$} & Non immunogenic \\
\hline & & Transient expression \\
\hline & & Re-administrable \\
\hline
\end{tabular}

\section{In vivo gene editing}

B

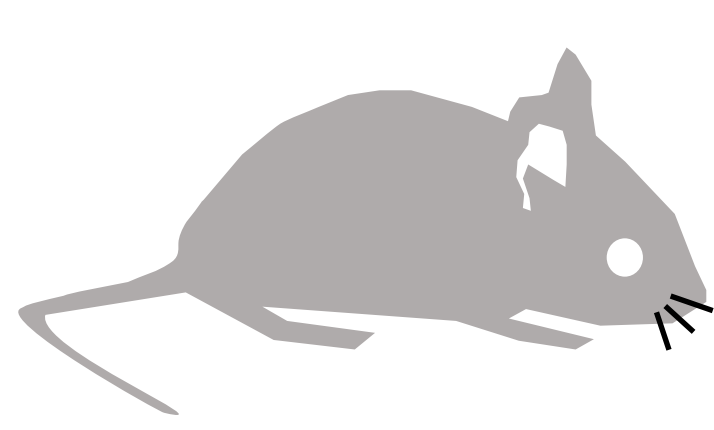

Wild-type mouse

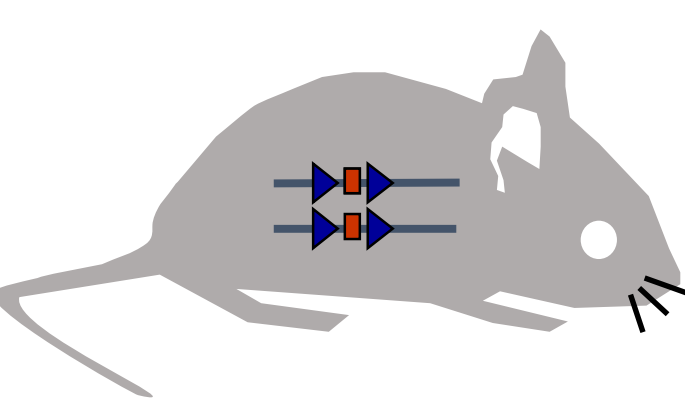

Pre-existing conditional and liver specific Cre/Lox mouse model

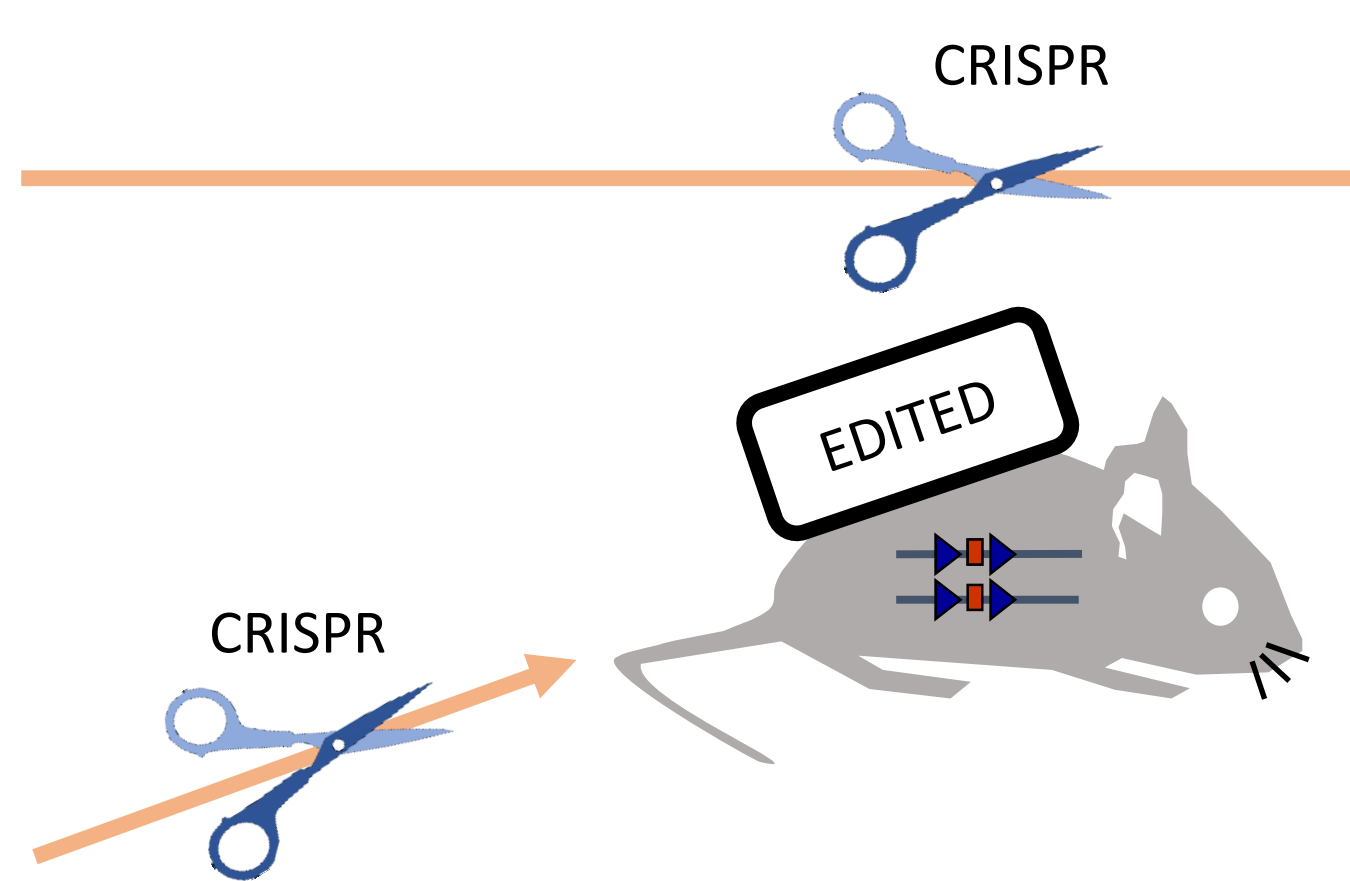

Sequential LOF using Cre/Lox mice
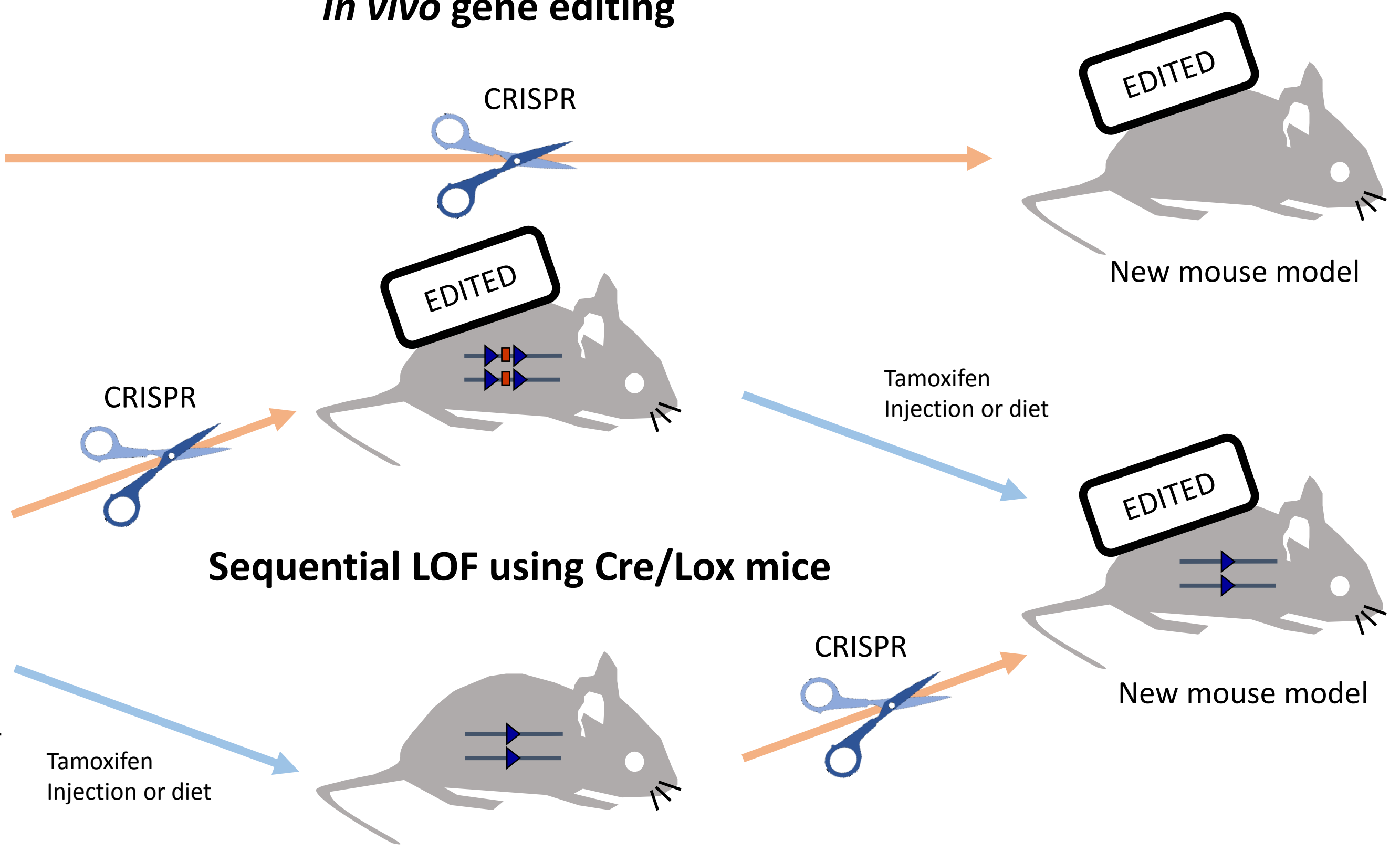

New mouse model

C
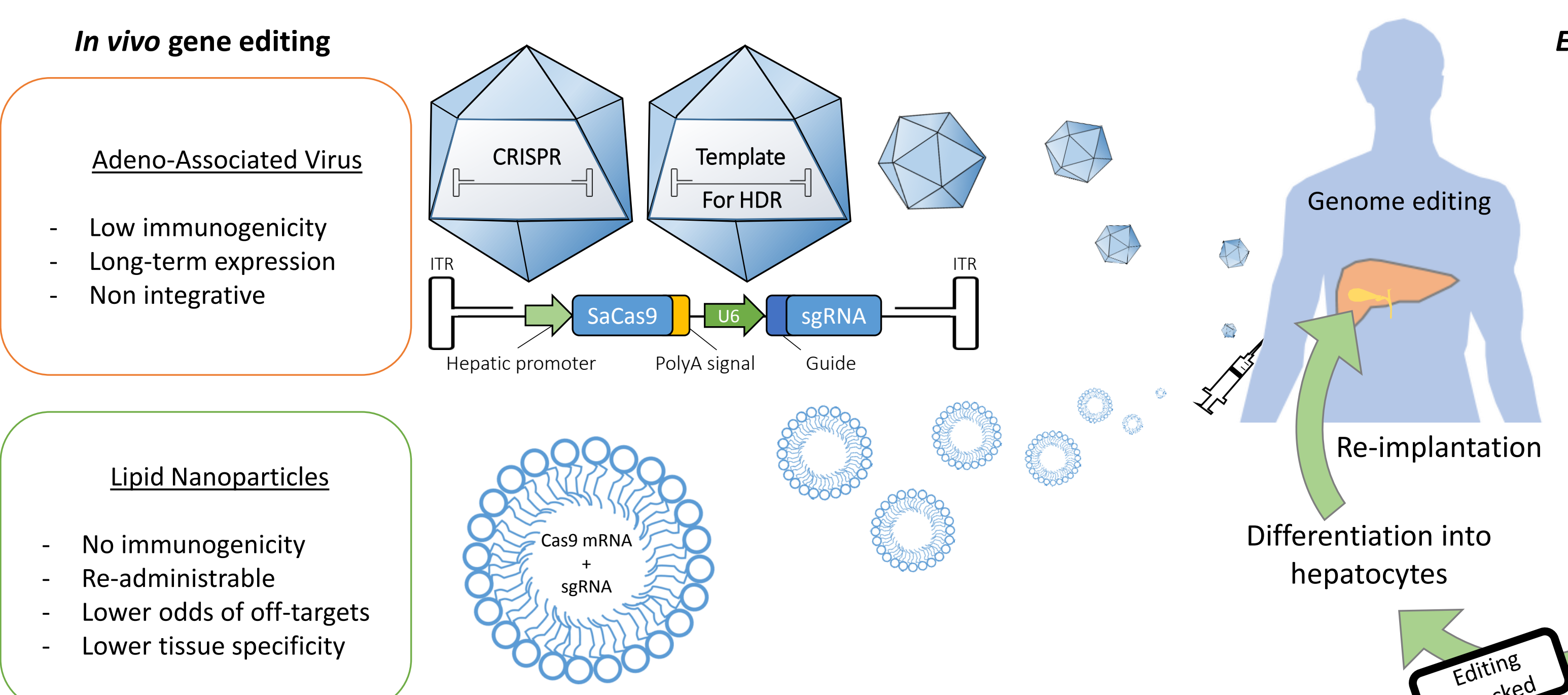

Ex vivo gene editing

Lipid Nanoparticles

No immunogenicity

Re-administrable

Lower odds of off-targets

Lower tissue specificity
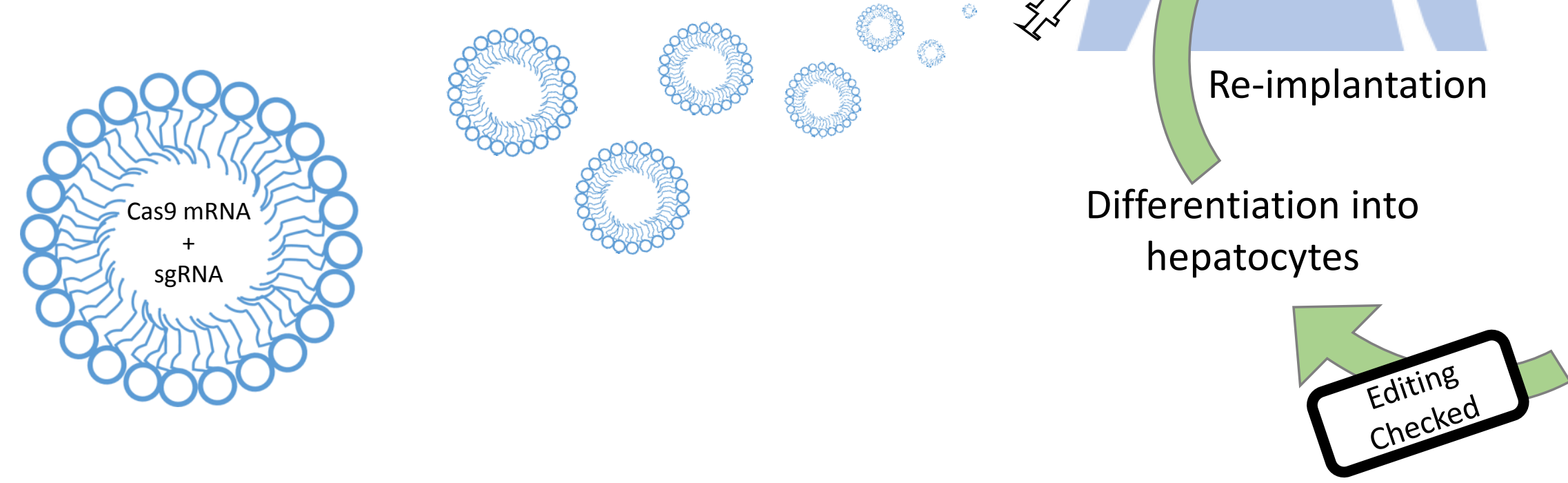

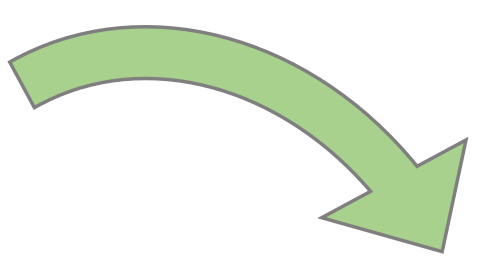

Induced Pluripotent Stem Cells

Gene Editing

Figure 2: Potential of CRISPR in liver research and therapy. A- In vivo gene editing toolbox recapitulating the different strategies available for mouse liver targeting. B- In vivo gene editing allows the rapid development of new mouse models and the sequential loss of function (LOF) of several genes if combined with already existing Cre/Lox mice. C- Potential strategies for genome engineering in clinics. Viral and non-viral delivery would allow direct in vivo gene editing in the liver (Left) while the use of induces pluripotent stem cells would allow ex vivo gene editing before re-implantation (Right). 